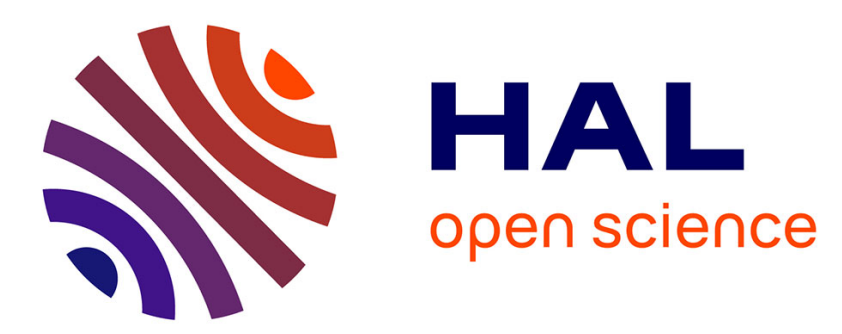

\title{
Nouvelle méthode de synthèse et propriétés de ferrite spinelle pour enregistrement magnétooptique réinscriptible
}

\author{
J. Ostoréro, H. Le Gall, M. Escorne, F. Soulette, A. Percheron-Guegan
}

\section{To cite this version:}

J. Ostoréro, H. Le Gall, M. Escorne, F. Soulette, A. Percheron-Guegan. Nouvelle méthode de synthèse et propriétés de ferrite spinelle pour enregistrement magnétooptique réinscriptible. Journal de Physique IV Proceedings, 1992, 02 (C3), pp.C3-23-C3-28. 10.1051/jp4:1992303 • jpa-00251508

\author{
HAL Id: jpa-00251508 \\ https://hal.science/jpa-00251508
}

Submitted on 1 Jan 1992

HAL is a multi-disciplinary open access archive for the deposit and dissemination of scientific research documents, whether they are published or not. The documents may come from teaching and research institutions in France or abroad, or from public or private research centers.
L'archive ouverte pluridisciplinaire HAL, est destinée au dépôt et à la diffusion de documents scientifiques de niveau recherche, publiés ou non, émanant des établissements d'enseignement et de recherche français ou étrangers, des laboratoires publics ou privés. 


\title{
Nouvelle méthode de synthèse et propriétés de ferrite spinelle pour enregistrement magnétooptique réinscriptible
}

\author{
J. OSTORÉRO, H. LE GALL"; M. ESCORNE, F. SOULETTE* et A. PERCHERON-GUEGAN \\ Laboratoire de Chimie Métallurgique des Terres Rares, CNRS, 1 Place A. Briand, 92195 Meudon cedex, \\ France \\ "Laboratoire de Magnétisme et Matériaux Magnétiques, CNRS, 1 Place A. Briand, 92195 Meudon cedex, \\ France
}

\begin{abstract}
Cobalt ferrite spinels films have been synthesized by oxidizing at different temperatures of a metallic alloy film $\mathrm{CoFe}_{2}$. This metallic film is deposited on glass substrates by sputtering of a $\mathrm{Fe}$ target covered by Co platelets. $\mathrm{X}$ ray diffraction data and electron microprobe analysis show that the spinel phase $\mathrm{CoFe}_{2} \mathrm{O}_{4}$ begins to form at $300{ }^{\circ} \mathrm{C}$ and crystallizes at higher temperatures. No preferential orientation was observed on glass substrates. Magnetic and magneto-optic properties (Faraday rotation (FR)) are a function of the oxidizing temperature $T_{0 x}$ and particularly at $632.8 \mathrm{~nm}$, FR are strongly related to the distribution of the $\mathrm{Co}^{2+}$ ions in the different sites of the spinel structure. Films prepared at $\mathrm{T}_{0 x}>600^{\circ} \mathrm{C}$ present properties close to those of bulk $\mathrm{CoFe}_{2} \mathrm{O}_{4}$. The high FR of the films indicate that this method could be used to synthesize Co ferrite spinel derived compounds as MO recording media.
\end{abstract}

Introduction:

Les spinelles ferrite de cobalt constituent des matériaux potentiels intéressants pour l'enregistrement magnéto-optique (MO) haute densité de l'information[1]-[3]. En effet, ils répondent aux principaux critères que nécessite cette technique: forte rotation Kerr ou Faraday[4], absorption optique et résistivité thermique suffisantes pour un échauffement local, composition et structure stables même après de nombreux cycles, matériaux de faible coût. Jusqu'à présent, ces composés ont été surtout étudiés sous forme de polycristaux ou monocristaux massifs. Nous présentons ici une nouvelle méthode de synthèse de films minces de ferrites par pulvérisation cathodique, plus particulièrement du ferrite de cobalt pur dont nous étudions les propriétés des films selon les conditions de préparation.

\section{Procédure expérimentale:}

A - Synthèse:

Les films minces de spinelles ferrites de cobalt $\mathrm{CoFe}_{2} \mathrm{O}_{4}$ sont préparés en 2 étapes selon la méthode suivante: On dépose tout d'abord un film d'alliage métallique Co-2Fe par copulvérisation cathodique d'une cible métallique constituée d'une mosaĩque de petites plaquettes de $\mathrm{Co}$ collèes sur une plaque de Fe pur. Le substrat dans toute cette étude est un verre Corning supportant des températures inférieures à $700^{\circ} \mathrm{C}$. Les conditions de synthèse du film précurseur par pulvérisation cathodique en atmosphère d'argon pur sont typiquement: pression de $210^{-1}$ à $610^{-1} \mathrm{~Pa}$ pour une puissance radiofréquence de 100 à $400 \mathrm{~W}$ (surface de la cible: $78 \mathrm{~cm}^{2}$ ). Dans une deuxième étape, les films d'alliage métallique ainsi obtenus sont oxydés à haute température en atmosphère contrôlée sous oxygène pur pour obtenir le film mince de ferrite spinelle. Cette technique présente l'avantage d'une grande souplesse de synthèse de compositions différentes en faisant varier la nature et/ou la surface de la mosaique métallique, ainsi que d'une vitesse de dépôt élevée par rapport à une pulvérisation cathodique d'oxydes. De plus, l'homogénéité très grande obtenue au niveau métallique est conservée pour l'oxyde. 


\section{$B$ - Caracténisation:}

La composition chimique des films préparés est déterminée par des analyses à la microsonde électronique $( \pm 2 \%$ ) et la structure spinelle vérifiée ainsi que la présence éventuelle d'autres phases par diffraction $X$. Les propriétés d'absorption optique sont déterminées à $300 \mathrm{~K}$ à l'aide d'un spectrophotomètre Cary de 240 à $3000 \mathrm{~nm}$. Les propriétés magnétiques sont mesuróes perpendiculairement au plan du film à $300 \mathrm{~K}$ à l'aide d'un magnétomètre à échantillon vibrant et de 40 à $300 \mathrm{~K}$ à l'aide d'un magnétomètre Manics DSM8 sous un champ magnétique variable jusqu'à $18 \mathrm{kOe}$. Les propriétés magnétooptiques (rotation Faraday) sont mesurées en employant une technique de modulation en fonction de la température $(75$ à $300 \mathrm{~K})$ et du champ magnétique $(0$ à $20 \mathrm{kOe})$.

\section{Résultats et interprétation:}

Apres oxydation, les films présentent une couleur marron par transparence devenant de plus en plus sombre lorsque l'épaisseur augmente. Ils adhèrent fortement sur le substrat de verre sauf si la copulvérisation du film métallique a été effectuée sous pression d'argon supérieure à 6 $10^{-1} \mathrm{~Pa}$. On observe alors souvent des craquelures après oxydation.

\section{1 - Diffraction $X$ :}

On a reporté sur la figure 1 (courbe a) le spectre de diffraction $X$ d'un film précuseur non traité. On constate la présence de 2 pics d'intensité faible dont la position correspond approximativement aux 2 raies intenses du Fe $\alpha$ (système cubique centré). Les analyses à la microsonde donnent la composition $\mathrm{CoFe}_{2}$; ceci est en accord avec les résultats concernant les alliages $\mathrm{Fe}-\mathrm{Co}$ qui cristallisent dans le système cubique centré\{5] en dessous de $985^{\circ} \mathrm{C}$ pour des teneurs en Co inférieures à $76 \%$. Après oxydation a $300^{\circ} \mathrm{C}$ sous oxygène, pendant $90 \mathrm{~h}$, on obtient le spectre de diffraction $X$ donné par la courbe b. On observe une phase mal cristallisée où les pics de l'alliage métallique ont fortement diminué d'intensité et l'apparition d'autres raies. Pour un film métallique oxydé à plus haute température, vers $600^{\circ} \mathrm{C}$ (courbe c), le spectre de diffraction $\mathrm{X}$ est celui d'une phase assez bien cristallisée. L'analyse à la microsonde indique une formule voisine de $\mathrm{CoFe}_{2} \mathrm{O}_{4}( \pm 2 \%)$. Afin de vérifier ces résultats, nous avons reporté l'emplacement théorique et les indices hkl des raies les plus intenses de ce composé[6]. On constate un excellent accord indiquant que le film de $\mathrm{CoFe}_{2}$ s'est bien oxydé en $\mathrm{CoFe}_{2} \mathrm{O}_{4}$. Les intensités des raies de diffraction du film proches de celles du polycristal massif montrent qu'il n'y a pas d'orientation préférentielle. Ceci est sans doute dû au substrat de verre et au fait que la cristallisation effectuée à $600^{\circ} \mathrm{C}$ n'a peut être pas permis la croissance et le grossissement de grains orientés

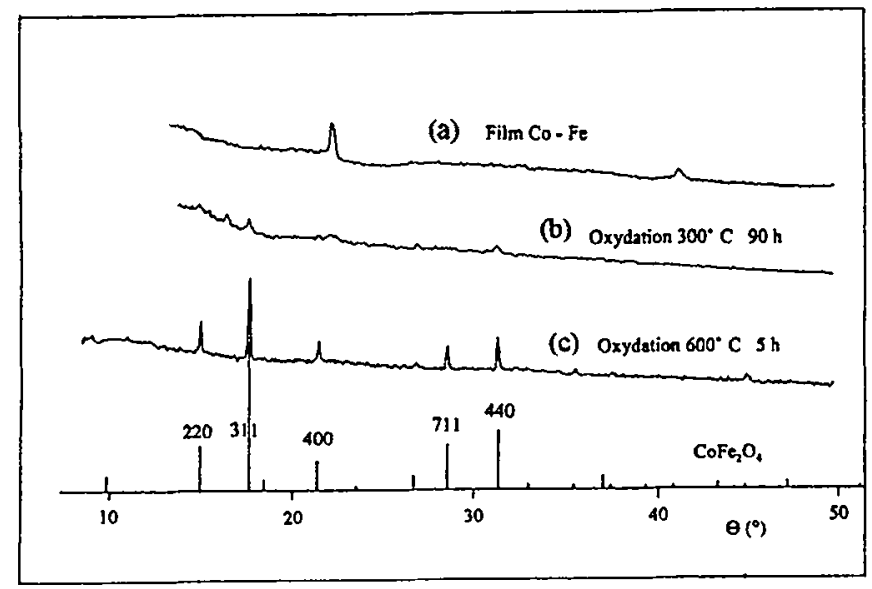

Figure 1 - Spectres de diffraction $X$

Pour des températures d'oxydation $\mathrm{T}_{\mathrm{ax}}$ intermédiaires entre 300 et $600^{\circ} \mathrm{C}$, les résultats de la microsonde et de diffraction X confirment la formation de la phase spinelle $\mathrm{CoFe}_{2} \mathrm{O}_{4}$. Lorsque $\mathrm{T}_{\text {ox }}$ augmente, il y a simultanément une diminution de la largeur des raies et un faible déplacement du 
spectre vers les $\theta$ plus grands. Pour $T_{o x}>600^{\circ} \mathrm{C}$, les spectres de diffraction $\mathrm{X}$ et les propriétés des films ne présentent plus de variations notables.

\section{2 - Spectres d'absorption optique:}

On a reporté sur la figure 2, les spectres d'absorption de 3 films de $\mathrm{CoFe}_{2} \mathrm{O}_{4}$ préparés à partir de la même expérience de pulvérisation, de même épaisseur, oxydés à 3 températures différentes: 400,500 , et $600{ }^{\circ} \mathrm{C}$. La contribution du substrat de verre a été soustraite dans les 3 cas. On constate que l'absorption optique très forte en dessous de $500 \mathrm{~mm}$ décroît ensuite rapidement aux longueurs d'onde plus grandes. Pour $\lambda<500 \mathrm{~nm}$, les 3 spectres présentent le même front d'absorption sans détail particulier. Dans la zone 1200-1800 nm, on note la présence d'une large bande d'absorption attribuée à $\mathrm{Co}^{2+}$ situé en site tétraédrique de la structure spinelle[7]. Cette bande diminue d'intensité lorsque la température d'oxydation augmente. Ce résultat et les expériences de diffraction $\mathrm{X}$ précédentes indiquent que lorsque $\mathrm{T}_{\text {ox }}$ augmente de $400 \mathrm{à} 600^{\circ} \mathrm{C}$, les films de ferrite spinelle $\mathrm{CoFe}_{2} \mathrm{O}_{4}$ ainsi préparés présentent une meilleure cristallinité avec des grains de tailles plus faibles et que le degré d'inversion du spinelle augmente $\left(\mathrm{CoFe}_{2} \mathrm{O}_{4}\right.$ massif présente une structure quasi inverse[4]).

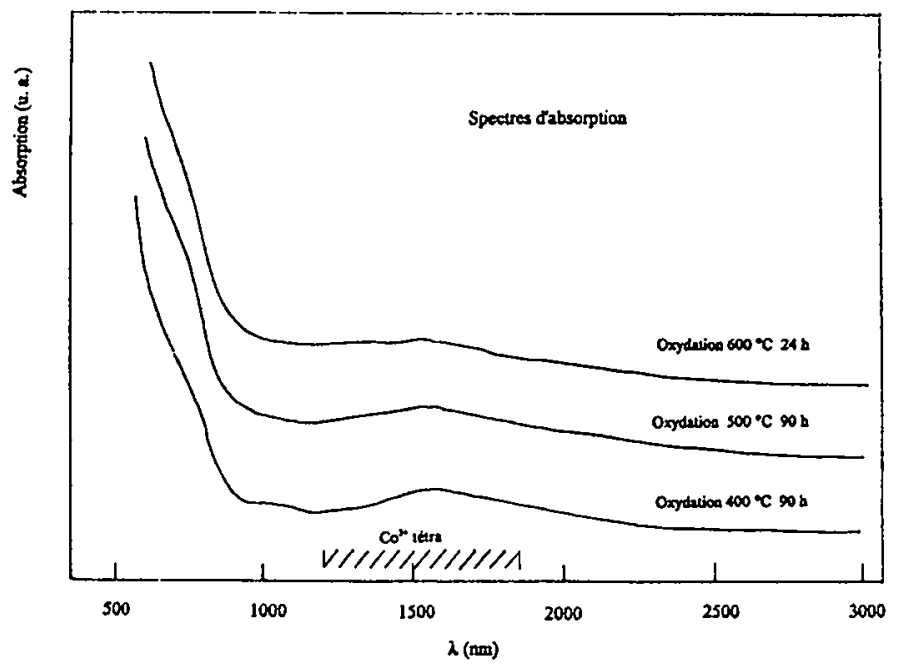

Figure 2 - Spectres d'absorption optique

\section{3 - Mesures magnétiques:}

La figure 3 représente les cycles d'hystérésis mesurés à la température ambiante de différents films. Le cycle d'hystérésis du film métallique (Fig. 3a) est très différent de celui obtenu sur les films oxydés (Fig. $3 \mathrm{~b}$, c, et d): $\mathrm{H}_{\mathrm{c}}$ voisin de 200 Oe et saturation magnétique pour des champs inférieurs à 1 kOe. La forme arrondie des cycles des films oxydés est due en partie à l'absence d'orientation préférentielle des couches. Le champ coercitif des films préparés à une même température $T_{\text {ox }}$ diminue lorsque l'épaisseur augmente: $H_{c}$ peut ainsi passer de $2200 \mathrm{Oe}$ à 700 Oe lorsque l'épaisseur du film passe respectivement de $0,54 \mu \mathrm{m}$ à $1,4 \mu \mathrm{m}$ (Fig. $3 \mathrm{~b}$ et d). Cette forte variation peut s'expliquer en partie par la formation de grains de dimensions plus importantes[8] dans le cas du film d'épaisseur plus grande. De même, les films synthétisés à $T_{o x}$ plus faibles, présentent pour la même épaisseur, un $\mathrm{H}_{c}$ plus faible (Fig. $3 c$ et d) lié à une taille de grains plus grande mise en évidence précédemment par les spectres de diffraction $X$. Le moment magnétique des films varie peu lorsque $T_{\text {ox }}$ passe de 400 à $600^{\circ} \mathrm{C}$ (Fig. 3c et d) et il est voisin de celui de $\mathrm{CoFe}_{2} \mathrm{O}_{4}$ massif. 

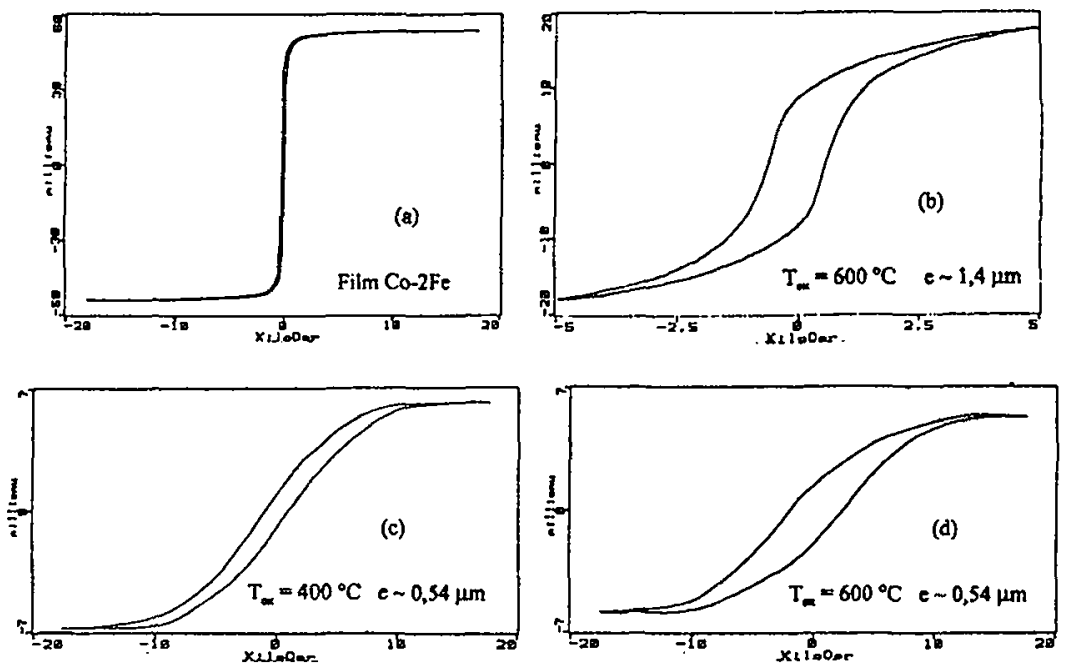

Figure 3 - Cycles d'hystérésis magnétique

\section{4- Propriétés magnécooptiques:}

On a mesuré à $300 \mathrm{~K}$ pour 3 tongueurs d'onde différentes $(543.5,632.8$, et $1152 \mathrm{~nm})$, la rotation Faraday $(\mathbf{R F})$ de films oxydés à des températures différentes, de même épaisseur $(\sim 0,54 \mu \mathrm{m})$ et issus de la même préparation par copulvérisation cathodique. Les résultats présentés sont obtenus après soustraction de la contribution du substrat de verre. En ce qui concerne les films de $\mathrm{CoFe}_{2} \mathrm{O}_{4}$ les mieux cristallisés $\left(600{ }^{\circ} \mathrm{C}\right)$, on note une très forte rotation Faraday: $2.510^{4}, 910^{3}$, et $-610^{3} \% \mathrm{~cm}$ pour respectivement $543.5,632.8$ et $1152 \mathrm{~nm}$. Les variations les plus importantes en fonction de $T_{\text {ox }}$ sont obtenues pour $\lambda=632.8 \mathrm{~mm}$ : On observe qu'effectivement, la RF des films de $\mathrm{CoFe}_{2} \mathrm{O}_{4}$ est fortement négative pour $\mathrm{T}_{\mathrm{ox}}=400^{\circ} \mathrm{C}$ (Fig. 4a), voisine de 0 dans la limite de précision des mesures pour $\mathrm{T}_{\mathrm{ox}}=500$ ${ }^{\circ} \mathrm{C}$ (Fig. 4b) et tres fortement positive pour $\mathrm{T}_{\mathrm{ox}}=600^{\circ} \mathrm{C}$ (Fig. $4 \mathrm{c}$ ). Pour $\mathrm{T}_{\mathrm{ox}}=500^{\circ} \mathrm{C}$, la $\mathrm{RF}$ s'annule indépendamment de l'aimantation. Il s'agit donc dans ce cas d'un point de compensation MO. Ceci a été vérifé également en mesurant une RF non nulle de ce film à $300 \mathrm{~K}$ pour $543.5 \mathrm{~nm}$ (Fig. 4d) et à 632.8 $\mathrm{nm}$ pour $\mathrm{T}<300 \mathrm{~K}$. Ces résultats sont à mettre en parallèle avec les variations du degré d'inversion des films de $\mathrm{CoFe}_{2} \mathrm{O}_{4}$ lorsque $\mathrm{T}_{\mathrm{ox}}$ varie. Crossley et al. [9] ont montré que la rotation Faraday $\Phi$ d'un composé ferrimagnétique est la somme des contributions $\mathrm{MO}$ des sous réseaux:

$$
\Phi=\Sigma_{l} \alpha_{i} M_{i}
$$

où $\alpha_{i}$ est le coefficient MO du sous réseau d'aimantation $\mathrm{M}_{i}$. Dans le cas des films de $\mathrm{CoFe}_{2} \mathrm{O}_{4}$ synthétisés pour $T_{o x}$ compris entre 400 et $600^{\circ} \mathrm{C}$, en première approximation, le degré d'inversion du spinelle a peu d'influence sur l'aimantation. Les très fortes variations de la rotation Faraday observées à $632.8 \mathrm{~nm}$ sont donc liées vraisemblablement à une contribution négative de $\mathrm{Co}^{2+}$ en site tétraédrique. 

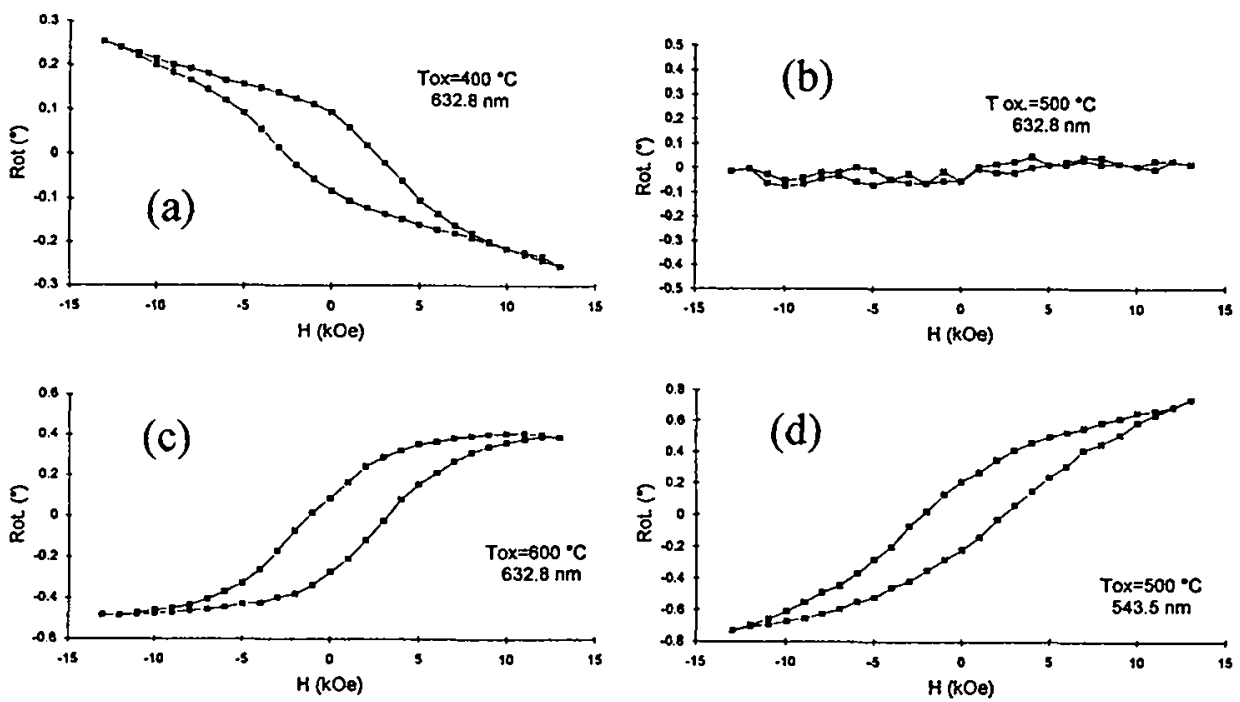

Figure 4 - Variation de la rotation Faraday

On a reporté sur la figure 5, les variations de l'aimantation et de la rotation Faraday en fonction de la température d'un film de $\mathrm{CoFe}_{2} \mathrm{O}_{4}$ de $1,5 \mu \mathrm{m}$ d'épaisseur $\left(\mathrm{T}_{\mathrm{ox}}=650^{\circ} \mathrm{C}\right)$. Les résultats obtenus sont en bon accord avec ceux mesurés sur $\mathrm{CoFe}_{2} \mathrm{O}_{4}$ massif [4], [10].

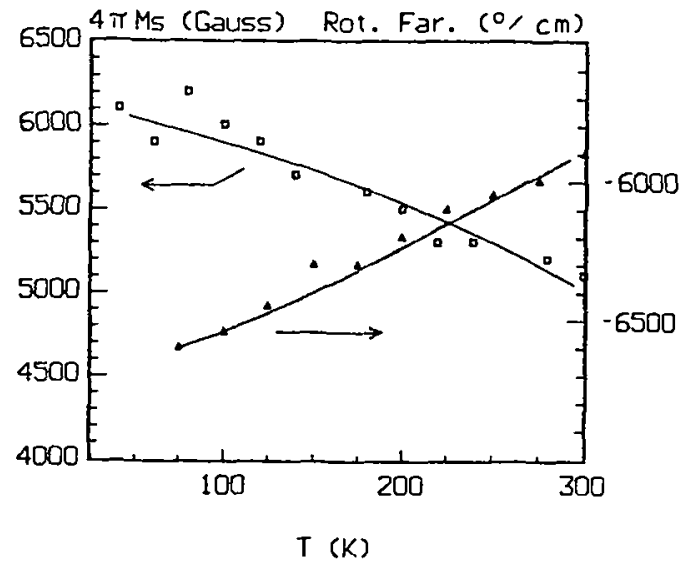

Figure 5 - Variation de l'aimantation et de la rotation Faraday en fonction de la température

Conclusion:

Nous avons réussi à synthétiser pour la première fois des films de $\mathrm{CoFe}_{2} \mathrm{O}_{4}$ par une méthode originale basée sur la copulvérisation cathodique d'un alliage métallique $\mathrm{C} 0-2 \mathrm{Fe}$ formant un film homogène précurseur qui est ensuite oxydé à haute température. Lorsque la température d'oxydation est supérieure à $400^{\circ} \mathrm{C}$ environ, les propriétés cristallographiques et magnétiques des films oxydés sont proches de celles de $\mathrm{CoFe}_{2} \mathrm{O}_{4}$ massif. La rotation Faraday de ces films met en évidence l'existence d'un point de compensation magnétooptique situé vers $300 \mathrm{~K}$ à $632.8 \mathrm{~nm}$ lié au degré d'inversion de la phase 
spinelle. Les valeurs de RF mesurés dépendent très fortement de ce degrè d'inversion. Ceci explique peut être les écarts importants relevés dans la littérature [1], [11]concernant la rotation Faraday de $\mathrm{CoFe}_{2} \mathrm{O}_{4}$ au voisinage de cette longueur d'onde. Dans le cas d'applications pour l'enregistrement MO de l'information, l'obtention de cycles d'hystérésis carrés peut être effectuée par des variations de la stoechiométrie du spinelle et/ou des traitements thermiques [3], ou par dopage d'autres éléments comme $\mathrm{Al}$ ou $\mathrm{Rh}$ [1], [2]. Cette méthode reste tout à fait adaptée à la préparation de ces films et peut s'étendre à d'autres composés oxydes et plus particulièrement ceux dont les alliages métalliques sont homogènes, cette homogénéité se retrouvant lors de l'oxydation.

Références:

[1] K. Suzuki, T. Namikawa, et Y. Yamazaki, Jpn. J. Appl. Phys. 27, 361 (1988)

[2] J. W. D. Martens et A. B. Voermans, I.E.E.E. Trans. Mag., 20, 1007 (1984)

[3] N.N. Evtihiev, N.A. Economov, A.R. Krebs, N.A. Zamjatina, V.D. Baurin, et V.G. Pinko, I.E.E.E. Trans. Mag., MAG-12, 773 (1976)

[4] J. Ostoréro, M. Guillot, M. Leblanc, et D. Rouet, J. Magn. Magn. Mater., 104-107, 425 (1992)

[5] R.M. Bozorth, "Ferrimagnetism", Van Nostrand Company, (1968)

[6] Fiche ASTM n 22-1086 et N.B.S., Mono. 25, Sec. 9 (1971)

[7] W.L. Peeters et J.W.D. Martens, J. Appl. Phys., 53, 8178 (1982)

[8] C. E. Davies, R.E. Somekh, J.E. Evetts, et P.A. Storey, I.E.E.E. Trans. Mag., 24, 2790 (1988)

[9] W.A. Crossley, R.W. Cooper, J.L. Page, R.P. Van Stapele, Phys. Rev., 181, 896 (1969)

[10] M. Guillot, J. Ostoréro, et A. Marchand, Z. Phys. B71, 193 (1988)

[11] M. Abe et M. Gomi, J. Magn. Magn. Mater., 84, 222 (1990) 